\title{
BMJ Open Association between highly active antiretroviral therapy and selected cardiovascular disease risk factors in sub-Saharan Africa: a systematic review and meta-analysis protocol
}

\author{
Christian Akem Dimala, ${ }^{1,2}$ Hannah Blencowe ${ }^{2}$
}

To cite: Dimala CA, Blencowe H. Association between highly active antiretroviral therapy and selected cardiovascular disease risk factors in subSaharan Africa: a systematic review and meta-analysis protocol. BMJ Open 2017;7: e013353. doi:10.1136/ bmjopen-2016-013353

- Prepublication history for this paper is available online. To view these files please visit the journal online (http://dx.doi.org/10.1136/ bmjopen-2016-013353).

Received 6 July 2016 Revised 14 February 2017 Accepted 15 February 2017



${ }^{1}$ Health and Human Development $(2 \mathrm{HD})$ research group, Douala, Cameroon ${ }^{2}$ Department of Infectious Disease Epidemiology, London School of Hygiene and Tropical Medicine, London, UK

Correspondence to Dr Christian Akem Dimala; Christian.Akem.Dimala1@ alumni.Ishtm.ac.uk

\begin{abstract}
Introduction: The increasing highly active antiretroviral therapy (HAART) coverage in sub-Saharan Africa (SSA) has been associated with increasing cardiovascular disease (CVD) incidence. However, the epidemiology of the association between HAART and CVD risk factors in SSA is sparse. We aim to assess the extent to which HAART is associated with selected cardiovascular risk factors (hypertension, diabetes, dyslipidaemia and metabolic syndrome) in SSA.
\end{abstract}

Methods and analysis: This will be a systematic review and meta-analysis of published studies on the association between HAART and CVD risk factors retrieved from Medline, Embase, Popline, Africa-Wide Information, African Index Medicus and the Cochrane library databases. Studies will be screened for eligibility according to the selection criteria by two independent reviewers. Eligible studies will be assessed for the quality of their evidence and risk of bias using the Quality Assessment Tool for Observational Cohort and Cross-Sectional Studies of the National Health Institute and the Grading of Recommendations Assessment, Development and Evaluation (GRADE) approach, with respect to the measured outcomes (hypertension, diabetes, dyslipidaemia and metabolic syndrome). A data abstraction form will be produced on Epi info V.7 and data analysis done on STATA V.14 statistical software. Summary estimates of measures of effects for the association between HAART use and the outcomes will be derived. Random effects meta-analyses will be performed and $\mathrm{I}^{2}$ statistic used to assess for heterogeneity between studies with respect to measured parameters. Qualitative synthesis will be used where data is insufficient to produce quantitative synthesis.

Ethics and dissemination: The protocol has been reviewed by the Research Governance \& Integrity Office of the Research Ethics Committee of the London School of Hygiene and Tropical Medicine and confirmed as not requiring ethical approval. The findings of this study will be made widely available especially to national HIV/AIDS committees formulating HIV/AIDS guidelines for their respective settings.

Trial registration number: CRD42016042306; Pre-results.

\section{Strengths and limitations of this study}

- This review will provide a summary of published studies on the extent to which antiretroviral therapy is associated to cardiovascular disease risk factors (hypertension, diabetes, dyslipidaemia and metabolic syndrome).

- The findings of this review will be very important to authorities involved in health policy formulation in the HIV/AIDS domain especially as several African countries are currently aligning to the WHO recommendations of test and treat for HIV/AIDS with massive HAART scale-up.

- This study will be limited to sub-Saharan Africa; however, this is the region in the world with the greatest HIV/AIDS burden.

\section{INTRODUCTION}

The past decade has seen the rapid increase in highly active antiretroviral therapy (HAART) coverage worldwide with about 15 million patients with HIV/AIDS having access to treatment in 2014, and more than $90 \%$ of them being from low-income and middle-income countries most affected by HIV/AIDS. ${ }^{1}$ In 2014, HAART coverage stood at $41 \%$ in Africa, however, HAART has been increasingly associated with cardiovascular disease (CVD).$^{2-7}$ Several mechanisms have been proposed to explain this association among which prolonged HAART use predisposing to atherosclerosis and major cardiovascular risk factors such as diabetes mellitus (DM) and hypertension ${ }^{5}{ }^{8}{ }^{8-11}$ which consequently result in CVD. Even though, earlier studies already suggested a probable association between HAART and CVD, Bloomfield et $a t^{2}$ in a systematic review of literature found that CVD appeared to be more frequent in HIV-infected populations in lowincome and middle-income countries. This is 
particularly important since the greatest increase in HAART coverage has been in these countries. ${ }^{1}$ In subSaharan Africa (SSA), this association between HAART and CVD has been confounded by the epidemiological transition characterised by the increased prevalence and incidence of non-communicable diseases due to changes in lifestyle patterns. On the other hand, there have been contrary reports of no association between exposure to HAART and CVD risk factors. ${ }^{12-15}$ Understanding the overall effect of HAART on CVD risk is therefore of public health importance since the continuous HAART scale-up has to be accompanied by appropriate guidelines and measures to ensure the long-term morbidity and mortality of these patients is not negatively affected, especially in low-income and middle-income countries with health systems inadequately equipped to manage these chronic conditions. In a recent systematic review by Dillon et $a l^{16}$ on the association between HIV, HAART and cardiometabolic traits in the SSA population, there was a difference in these traits between HIV-infected patients and uninfected individuals and between HAART-treated and HAART- naïve patients, suggesting a probable effect of HAART. However, this review compared the standardised mean differences in blood pressure, blood glucose and lipid profile parameters between the groups and not actually on hypertension, diabetes or dyslipidaemia, respectively. Moreover, more recent studies have been published since then comparing prevalence and incidence of these risks factors in HAART-treated and HAART-naïve patients. We therefore aim to systematically review, and, where appropriate, perform a meta-analysis of the published studies on the extent to which exposure to HAART is associated to these CVD risk factors in SSA (figure 1).

\section{Research question}

1. Is there an association between HAART and selected cardiovascular risk factors (hypertension, diabetes, dyslipidaemia and metabolic syndrome) in patients with HIV/AIDS in SSA?

\section{Research objectives}

1. To estimate overall measures of effect for the association between HAART and hypertension in patients with HIV/AIDS in SSA.

2. To estimate overall measures of effect for the association between HAART and diabetes in these patients.

3. To estimate overall measures of effect for the association between HAART and dyslipidaemia in these patients.

4. To estimate overall measures of effect for the association between HAART and metabolic syndrome in these patients.

\section{METHODS}

Study research question design and eligibility criteria

This will be a systematic review and meta-analysis of published studies.
We will include:

- Cross-sectional, cohort, case-control and randomised controlled trials with data on the prevalence and/or incidence of hypertension, diabetes, lipid profile or metabolic syndrome in patients with HIV/AIDS;

- studies with comparable HAART-treated and HAARTnaïve populations;

- studies published in English and between January 1, 2003 and July 1, 2016 in the selected databases;

- studies involving participants aged 18 and above, living in one of the countries in SSA.

We will exclude:

- Unpublished manuscripts and conference abstracts;

- studies comparing the mean blood pressure, glucose and lipid levels in the HAART-treated and HAARTnaïve groups rather than the actual prevalence or incidence of hypertension, DM, dyslipidaemia and metabolic syndrome, respectively;

- studies with diagnostic criteria and cut-off values for hypertension, DM, abnormal lipid profiles and metabolic syndrome different from those internationally recognised;

- studies whose data will not be sufficient to calculate appropriate measures of effect;

- same studies published in different journals with the same or a different title.

For studies with several publications of their findings over time, the most recent of the studies will be chosen. For the meta-analysis, studies with a documented measure of effect on the selected cardiovascular risk factors comparing the HAART-treated and HAART-naive groups will be included. For studies without reported measures of effect, these will be computed if the provided data is adequate.

\section{Search strategy and identification of studies}

The following databases will be searched for eligible studies: Medline, Embase, Popline, Africa Wide Information, African Index Medicus and the Cochrane library. Medical subject headings $(\mathrm{MeSH})$ and free text searches will be used on Medline and Embase databases (table 1). Articles returned by the search will be saved to the Zotero V.4.0.29.10 software which will be used to remove duplicates. The titles and abstracts of the articles remaining after exclusion of duplicates will be assessed for eligibility according to the inclusion and exclusion criteria. Based on the year of introduction of HAART in SSA, the review will be focused on articles published between 2003 and 2016 .

The full text of all potentially eligible studies will then be reviewed by two independent reviewers (CAD and $\mathrm{HB}$ ), and any disagreement between reviewers with respect to eligible studies for inclusion in the analysis will be settled by a third reviewer. The reference lists of eligible studies and reviews will also be assessed for more eligible studies. A list of the potentially eligible studies excluded from the final analysis will be produced with the reasons for exclusion mentioned. A Preferred 
Figure 1 Conceptual framework showing the multiple CVD risk factors and the investigated association between HAART and the selected CVD risk factors. CVD, cardiovascular disease; HAART, highly active antiretroviral therapy.

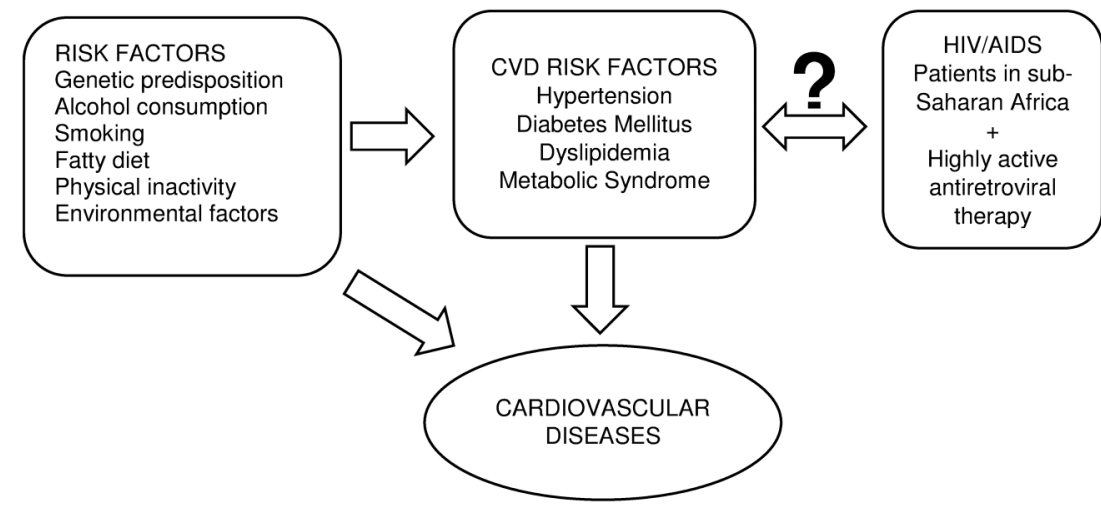

Reporting Items for Systematic Reviews and MetaAnalyses (PRISMA) flow chart detailing the number of articles identified, screened, included and excluded will be produced.

Data abstraction, data analysis and quality assessment A data abstraction form will be produced on Epi info V.7 statistical software and pretested by the principal investigator. The following data will be extracted from the selected studies: first author, publication year, study design and setting, duration of study, summary information of the socio-demographic and clinical details of the participants such as mean or median age, sex distribution, duration of HIV infection and of antiretroviral therapy, the measures of central tendency, disease frequency and effect for the selected cardiovascular risk factors in the HAART and HAART-naïve groups, statin use among participants, distinction between gardenvariety hyperlipidaemia and that resulting from probable HAART use and any other information considered relevant. The appropriate measures of effect will be derived in studies that provide sufficient data to do so.

Data will be analysed using STATA V.14 statistical software. Owing to the possibility of heterogeneity among the studies, random effects meta-analysis models will be preferentially reported over fixed-effects models.

For objectives 1, 2, 3 and 4, ORs comparing the HAART+ and HAART - groups will be calculated and

Table 1 Search strategy for the Medline, Embase and Africa-Wide Information for the review on the association between highly active antiretroviral therapy and selected cardiovascular disease risk factors in subSaharan Africa

\section{\# Search words}

1 Africa (MeSH terms) OR Africa OR Algeria OR Angola OR Benin OR Botswana OR Burkina Faso OR Burundi OR Cameroon OR Canary Islands OR Cape Verde OR Central African Republic OR Chad OR Comoros OR Congo OR Democratic Republic of Congo OR Djibouti OR Egypt OR Equatorial Guinea OR Eritrea OR Ethiopia OR Gabon OR Gambia OR Ghana OR Guinea OR Guinea Bissau OR Ivory Coast OR Cote dlvoire OR Jamahiriya OR Jamahiryia OR Kenya OR Lesotho OR Liberia OR Libya OR Libia OR Madagascar OR Malawi OR Mali OR Mauritania OR Mauritius OR Mayotte OR Morocco OR Mozambique OR Mocambique OR Namibia OR Niger OR Nigeria OR Principe OR Reunion OR Rwanda OR Sao Tome OR Senegal OR Seychelles OR Sierra Leone OR Somalia OR South Africa OR St Helena OR Sudan OR Swaziland OR Tanzania OR Togo OR Tunisia OR Uganda OR Western Sahara OR Zaire OR Zambia OR Zimbabwe OR Central Africa OR Central African OR West Africa OR West African OR Western Africa OR Western African OR East Africa OR East African OR Eastern Africa OR Eastern African OR North Africa OR North African OR Northern Africa OR Northern African OR South African OR Southern Africa OR Southern African OR subSaharan Africa OR subSaharan African OR subSaharan Africa OR subSaharan African

2 Antiretroviral therapy, highly active (MeSH terms) OR highly active antiretroviral therapy OR antiretroviral therapy OR antiretroviral OR HAART OR ART OR antiretroviral OR Nucleotide reverse transcriptase inhibitor OR NRTI OR Non-nucleoside reverse transcriptase inhibitor OR NNRTI OR Protease inhibitor OR PI OR PIs OR lopinavir OR ritonavir OR lamivudine OR zidovudine OR stavudine OR nevirapine OR efavirenz OR tenofovir OR emtricitabine OR atazanavir OR darunavir

3 Cardiovascular diseases (MeSH terms) OR hypertension OR high blood pressure OR systolic blood pressure OR diastolic blood pressure OR SBP OR DBP OR diabetes mellitus OR diabetes OR type 2 diabetes OR type 2 diabetes mellitus OR diabetic OR type 2 diabetic OR dysglycemia OR dysglycaemia OR hyperglycemia OR hyperglycaemia OR glucose OR insulin resistance OR insulin OR hyperinsulinemia OR hyperinsulinaemia OR dyslipidemias OR dyslipidemia OR hyperlipidemia OR hypercholesterolemia OR hypertriglyceridemia OR cholesterol OR triglyceride OR triglycerides OR HDL OR LDL OR VLDL OR hyperlipoproteinemia OR lipoprotein OR hyperlipidaemia OR hypercholesterolaemia OR hypertriglyceridaemia OR metabolic syndrome

4 \#1 AND \#2 AND \#3

MeSH, Medical Subject Headings. 
Table 2 Definition of outcomes per included studies

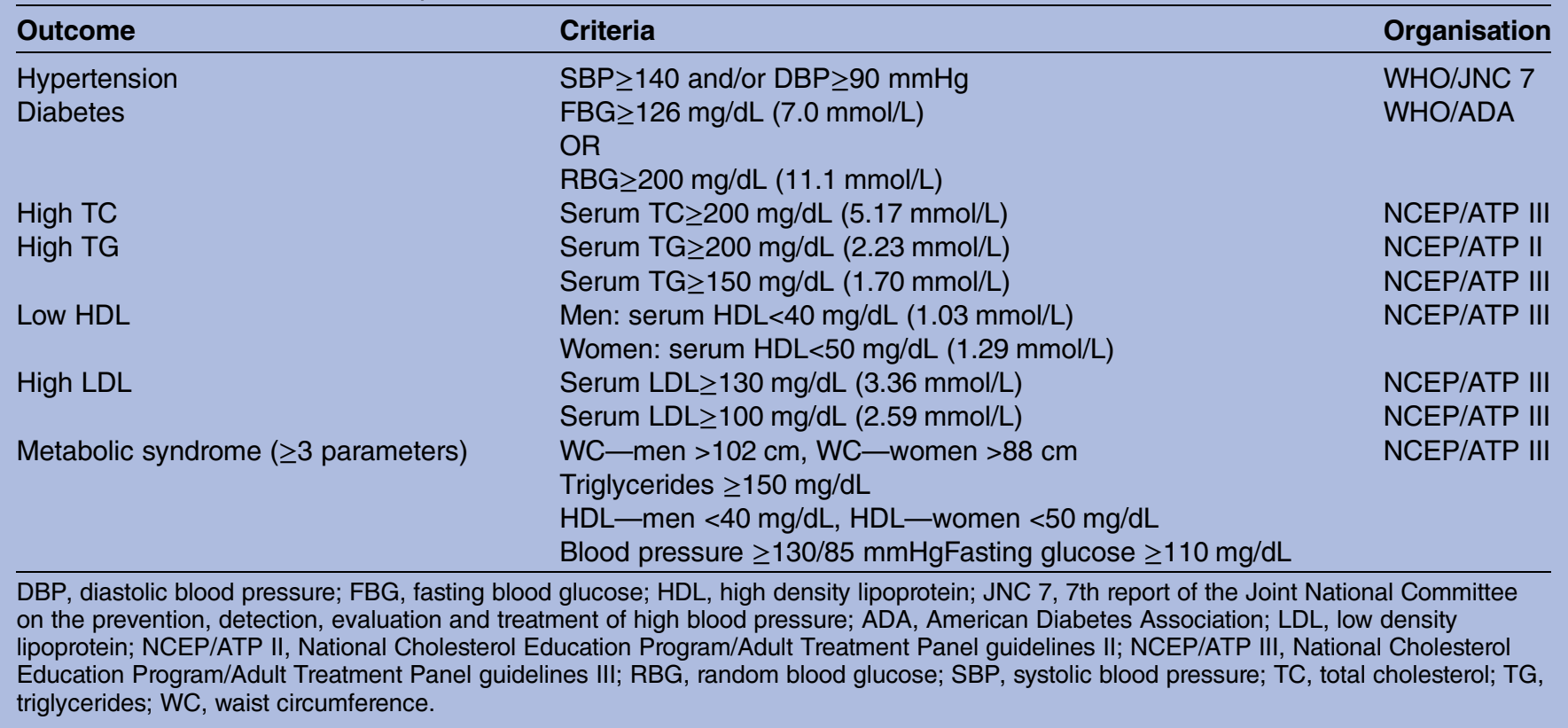

Table 3 Quality assessment tool for observational cohort and cross-sectional studies
1. Was the research question or objective in this paper clearly stated?
2. Was the study population clearly specified and defined?
3. Was the participation rate of eligible persons at least $50 \%$ ?
4. Were all the subjects selected or recruited from the same or similar populations (including the same time period)? Were inclusion and exclusion criteria for being in the study prespecified and applied uniformly to all participants?
5. Was a sample size justification, power description or variance and effect estimates provided?
6. For the analyses in this paper, were the exposure(s) of interest measured prior to the outcome
(s) being measured?
7. Was the timeframe sufficient so that one could reasonably expect to see an association between exposure and outcome if it existed?
8. For exposures that can vary in amount or level, did the study examine different levels of the exposure as related to the outcome (e.g., categories of exposure, or exposure measured as continuous variable)?
9. Were the exposure measures (independent variables) clearly defined, valid, reliable and
implemented consistently across all study participants?
10. Was the exposure(s) assessed more than once over time?
11. Were the outcome measures (dependent variables) clearly defined, valid, reliable and implemented consistently across all study participants?
12. Were the outcome assessors blinded to the exposure status of participants?
13. Was loss to follow-up after baseline $20 \%$ or less?
14. Were key potential confounding variables measured and adjusted statistically for their impact on the relationship between exposure(s) and outcome(s)?
Quality Rating (Good, fair or poor) (see guidance)
Rater \#1 initials:
Rater \#2 initials:
Additional Comments (If POOR, please state why):
$\mathrm{CD}$, cannot determine; NA, not applicable; NR, not reported.

Criteria

Other (CD,

summarised. The exposure variable will be HAART use and the outcome variable will be the respective cardiovascular risk factors: hypertension, diabetes, dyslipidaemia and metabolic syndrome. These outcomes will be defined according to the standard international criteria (table 2). Pooled estimates of measures of effect will be derived using random effects models, the Cochran's $Q$ test will be used to assess for evidence of between-study 
Table 4 PRISMA-P 2015 checklist for the study protocol of the systematic review on the association between highly active antiretroviral therapy and selected cardiovascular risk factors in subSaharan Africa

\begin{tabular}{|c|c|c|c|}
\hline Section and topic & $\begin{array}{l}\text { Item } \\
\text { no }\end{array}$ & Checklist item & Page \\
\hline \multicolumn{4}{|l|}{ Administrative information } \\
\hline \multicolumn{4}{|l|}{ Title } \\
\hline Identification & $1 \mathrm{a}$ & Identify the report as a protocol of a systematic review & 1 \\
\hline Update & $1 b$ & If the protocol is for an update of a previous systematic review, identify as such & NAP \\
\hline Registration & 2 & $\begin{array}{l}\text { If registered, provide the name of the registry (such as PROSPERO) and registration } \\
\text { number }\end{array}$ & 2 \\
\hline \multicolumn{4}{|c|}{ ( } \\
\hline Contact & $3 a$ & $\begin{array}{l}\text { Provide name, institutional affiliation, email address of all protocol authors; provide } \\
\text { physical mailing address of corresponding author }\end{array}$ & 1 \\
\hline Contributions & $3 b$ & Describe contributions of protocol authors and identify the guarantor of the review & 10 \\
\hline Amendments & 4 & $\begin{array}{l}\text { If the protocol represents an amendment of a previously completed or published } \\
\text { protocol, identify as such and list changes; otherwise, state plan for documenting } \\
\text { important protocol amendments }\end{array}$ & NAP \\
\hline \multicolumn{4}{|c|}{ p - } \\
\hline Sources & $5 a$ & Indicate sources of financial or other support for the review & 10 \\
\hline Sponsor & $5 b$ & Provide name for the review funder and/or sponsor & NAP \\
\hline Role of sponsor & $5 c$ & $\begin{array}{l}\text { Describe roles of funder(s), sponsor(s) and/or institution(s), if any, in developing the } \\
\text { protocol }\end{array}$ & NAP \\
\hline \multicolumn{4}{|c|}{ TIN } \\
\hline Rationale & 6 & Describe the rationale for the review in the context of what is already known & 4 \\
\hline Objectives & 7 & $\begin{array}{l}\text { Provide an explicit statement of the question(s) the review will address with reference } \\
\text { to participants, interventions, comparators and outcomes (PICO) }\end{array}$ & 5 \\
\hline \multicolumn{4}{|c|}{ 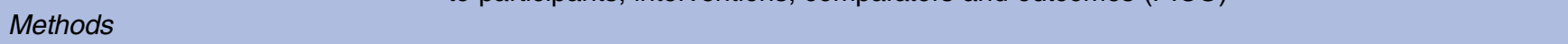 } \\
\hline Eligibility criteria & 8 & $\begin{array}{l}\text { Specify the study characteristics (such as PICO, study design, setting, time frame) } \\
\text { and report characteristics (such as years considered, language, publication status) to } \\
\text { be used as criteria for eligibility for the review }\end{array}$ & 5 \\
\hline Information sources & 9 & $\begin{array}{l}\text { Describe all intended information sources (such as electronic databases, contact with } \\
\text { study authors, trial registers or other grey literature sources) with planned dates of } \\
\text { coverage }\end{array}$ & 6 \\
\hline Search strategy & 10 & $\begin{array}{l}\text { Present draft of search strategy to be used for at least one electronic database, } \\
\text { including planned limits, such that it could be repeated }\end{array}$ & 11 \\
\hline \multicolumn{4}{|c|}{ 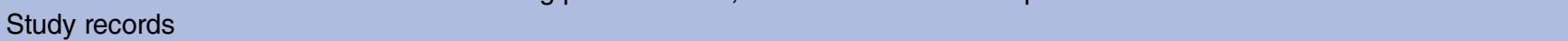 } \\
\hline \multirow[t]{3}{*}{$\begin{array}{l}\text { Data management } \\
\text { Selection criteria } \\
\text { Data collection }\end{array}$} & $11 a$ & $\begin{array}{l}\text { State the process that will be used for selecting studies (such as two independent } \\
\text { reviewers) through each phase of the review (that is, screening, eligibility and } \\
\text { inclusion in meta-analysis) }\end{array}$ & $6 \& 7$ \\
\hline & $11 b$ & $\begin{array}{l}\text { Describe planned method of extracting data from reports (such as piloting forms, } \\
\text { done independently or in duplicate), any processes for obtaining and confirming data } \\
\text { from investigators }\end{array}$ & $6 \& 7$ \\
\hline & $11 \mathrm{c}$ & $\begin{array}{l}\text { List and define all variables for which data will be sought (such as PICO items, } \\
\text { funding sources), any preplanned data assumptions and simplifications }\end{array}$ & $6 \& 7$ \\
\hline Data items & 12 & & \\
\hline $\begin{array}{l}\text { Outcome and } \\
\text { prioritisation }\end{array}$ & 13 & $\begin{array}{l}\text { List and define all outcomes for which data will be sought, including prioritisation of } \\
\text { main and additional outcomes, with rationale }\end{array}$ & $6 \& 7$ \\
\hline $\begin{array}{l}\text { Risk of bias in individual } \\
\text { subjects }\end{array}$ & 14 & $\begin{array}{l}\text { Describe anticipated methods for assessing risk of bias of individual studies, } \\
\text { including whether this will be done at the outcome or study level, or both; state how } \\
\text { this information will be used in data synthesis }\end{array}$ & 7 \\
\hline \multirow[t]{4}{*}{ Data synthesis } & $15 a$ & Describe criteria under which study data will be quantitatively synthesised & 7 \\
\hline & $15 b$ & $\begin{array}{l}\text { If data are appropriate for quantitative synthesis, describe planned summary } \\
\text { measures, methods of handling data and methods of combining data from studies, } \\
\text { including any planned exploration of consistency (such as } 12 \text {, Kendall's ô) }\end{array}$ & 7 \\
\hline & $15 c$ & $\begin{array}{l}\text { Describe any proposed additional analyses (such as sensitivity or subgroup } \\
\text { analyses, metaregression) }\end{array}$ & 7 \\
\hline & $15 d$ & If quantitative synthesis is not appropriate, describe the type of summary planned & 7 \\
\hline Metabias(es) & 16 & $\begin{array}{l}\text { Specify any planned assessment of metabias(es) (such as publication bias across } \\
\text { studies, selective reporting within studies) }\end{array}$ & 7 \\
\hline $\begin{array}{l}\text { Confidence in cumulative } \\
\text { evidence }\end{array}$ & 17 & $\begin{array}{l}\text { Describe how the strength of the body of evidence will be assessed (such as } \\
\text { GRADE) }\end{array}$ & 7 \\
\hline
\end{tabular}


heterogeneity and the $\mathrm{I}^{2}$ test statistics will be used to assess the degree of heterogeneity among the studies. Corresponding forests plots will also be produced. The Harbord's test and the Peter's test ${ }^{17}$ will be used to statistically assess for funnel plot asymmetry and small-study effects with low $p$ values indicating evidence of plot asymmetry, small-study effect and possibly publication bias. Metaregressions and subgroup stratified analyses will be done according to important study characteristics such as: study quality, study location, sample size, adjusting of confounders or not, antiretroviral therapy agents and regimens, statin use and any other relevant parameters identified during the abstraction, to explore if these study characteristics could be potential sources of heterogeneity among the studies. A minimum of two studies per subgroup will be considered adequate for the stratified analyses. Qualitative synthesis will be used in cases where data extracted is insufficient to perform quantitative synthesis.

Eligible studies will be assessed for the quality of their evidence and risk of bias by two independent reviewers (CAD and $\mathrm{HB}$ ) with respect to: flaws in study design, comparability of the HAART+ and HAART- groups, inadequately measured or managed confounders, determination of associations at univariate or multivariate levels of analysis, inaccuracies in the measurements of the key parameters such as hypertension, diabetes, lipid profile and metabolic syndrome or inappropriate diagnostic cut-off values. The Quality Assessment Tool for Observational Cohort and Cross-Sectional Studies of the National Health Institute/National Heart, Lung, and Blood Institute (table 3) which takes into account all these parameters will be used to grade the overall quality of each individual study as good, fair or poor.

The Grading of Recommendations Assessment, Development and Evaluation (GRADE) approach will be used to assess for quality of evidence provided by the included studies with respect to the measured outcomes (hypertension, diabetes, dyslipidaemia and metabolic syndrome), by the two reviewers (CAD and $\mathrm{HB}$ ) taking into account the study limitations, inconsistencies, indirectness, imprecision and publication bias. Overall quality of evidence following assessment will be graded as high, moderate, low or very low.

\section{Reporting and amendments to protocol}

The PRISMA-P guidelines for the reporting of systematic reviews and meta-analysis protocols have been used for this systematic review protocol as illustrated on table 4 . The completed report will be reported in accordance with the PRISMA guidelines using the PRISMA checklist. Amendments will be made to the study if and only if necessary and will be clearly documented and justified.

\section{CONCLUSION}

There still remains contradictory evidence on this association between HAART and CVD in SSA. As such, this review will present a summary of what is documented in SSA as far as CVD epidemiology and HIV/AIDS is concerned. This is of utmost importance since many SSA countries are currently aligning to the WHO recommendations of test and treat for HIV/AIDS. This massive HAART scale-up could be faced with rather increasing morbidity and mortality from CVDs in patients with HIV/AIDS, if these underlying mechanisms are not well understood and measures such as concurrent scale-up of CVD screening and treatment services envisaged.

\section{ETHICAL CONSIDERATIONS AND DISSEMINATION}

The Research Governance \& Integrity Office of the Research Ethics Committee of the London School of Hygiene and Tropical Medicine reviewed the study and affirmed no ethical approval is required. The findings of this study will be made widely available to the appropriate authorities involved in health policy formulation especially with respect to HIV/AIDS treatment guidelines and also to the scientific community for subsequent updating as more recent studies become available.

Contributors $C A D$ and $H B$ conceived and designed the experiments. $C A D$ produced the manuscript. HB reviewed the manuscript. Both authors approved the final copy of the manuscript.

Competing interests None declared.

Provenance and peer review Not commissioned; externally peer reviewed.

Open Access This is an Open Access article distributed in accordance with the Creative Commons Attribution Non Commercial (CC BY-NC 4.0) license, which permits others to distribute, remix, adapt, build upon this work noncommercially, and license their derivative works on different terms, provided the original work is properly cited and the use is non-commercial. See: http:// creativecommons.org/licenses/by-nc/4.0/

\section{REFERENCES}

1. Joint United Nations Programme on HIV/AIDS. Global AIDS Update 2016. UNAIDS, 2016

2. Bloomfield GS, Khazanie P, Morris A, et al. HIV and noncommunicable cardiovascular and pulmonary diseases in low-and middle-income countries in the ART era: what we know and best directions for future research. J Acquir Immune Defic Syndr 2014;67:S40-53.

3. Carr A, Samaras K, Thorisdottir A, et al. Diagnosis, prediction, and natural course of HIV-1 protease-inhibitor-associated lipodystrophy, hyperlipidaemia, and diabetes mellitus: a cohort study. Lancet 1999;353:2093-9.

4. Behrens G, Dejam A, Schmidt $H$, et al. Impaired glucose tolerance, beta cell function and lipid metabolism in HIV patients under treatment with protease inhibitors. AIDS 1999;13:F63-70.

5. Cattelan AM, Trevenzoli M, Sasset $L$, et al. Indinavir and systemic hypertension. AIDS 2001;15:805-7.

6. Brown TT, Cole SR, Li X, et al. Antiretroviral therapy and the prevalence and incidence of diabetes mellitus in the multicenter AIDS cohort study. Arch Intern Med 2005;165:1179-84.

7. Holmberg SD, Moorman AC, Williamson JM, et al. Protease inhibitors and cardiovascular outcomes in patients with HIV-1. Lancet 2002;360:1747-8.

8. Dimala CA, Atashili J, Mbuagbaw JC, et al. Prevalence of hypertension in HIV/AIDS patients on highly active antiretroviral therapy (HAART) compared with HAART-naïve patients at the Limbe Regional Hospital, Cameroon. PLOS ONE. 2016;11: e0148100.

9. Ekali LG, Johnstone LK, Echouffo-Tcheugui JB, et al. Fasting blood glucose and insulin sensitivity are unaffected by HAART duration in Cameroonians receiving first-line antiretroviral treatment. Diabetes Metab 2013;39:71-7. 
10. Murata $\mathrm{H}, \mathrm{Hruz} \mathrm{PW}$, Mueckler M. The mechanism of insulin resistance caused by HIV protease inhibitor therapy. J Biol Chem 2000;275:20251-4.

11. Samaras K. Prevalence and pathogenesis of diabetes mellitus in HIV-1 infection treated with combined antiretroviral therapy: JAIDS $J$ Acquir Immune Defic Syndr 2009;50:499-505.

12. Thiébaut $\mathrm{R}, \mathrm{El}-\mathrm{Sadr}$ WM, Friis-Moller $\mathrm{N}$, et al. Predictors of hypertension and changes of blood pressure in HIV-infected patients. Antivir Ther 2005;10:811.

13. Jung $\mathrm{O}$, Bickel M, Ditting $\mathrm{T}$, et al. Hypertension in HIV-1-infected patients and its impact on renal and cardiovascular integrity. Nephrol Dial Transplant 2004;19:2250-8.

14. Dave JA, Lambert EV, Badri M, et al. Effect of nonnucleoside reverse transcriptase inhibitor-based antiretroviral therapy on dysglycemia and insulin sensitivity in South African HIV-infected patients. J Acquir Immune Defic Syndr 2011;57:284-9.

15. Manuthu EM, Joshi MD, Lule GN, et al. Prevalence of dyslipidemia and dysglycaemia in HIV infected patients. East Afr Med J 2008;85:10-17.

16. Dillon DG, Gurdasani D, Riha J, et al. African Partnership for Chronic Disease Research (APCDR). Association of HIV and ART with cardiometabolic traits in sub-Saharan Africa: a systematic review and meta-analysis. J Epidemiol 2013;42:1754-71.

17. Sterne JAC, Egger M, Moher D. Chapter 10: addressing reporting biases. In: Higgins JPT, Green S, eds. Cochrane handbook for systematic reviews of interventions. Chichester, UK: John Wiley \& Sons, Ltd, 2008:314-19. 